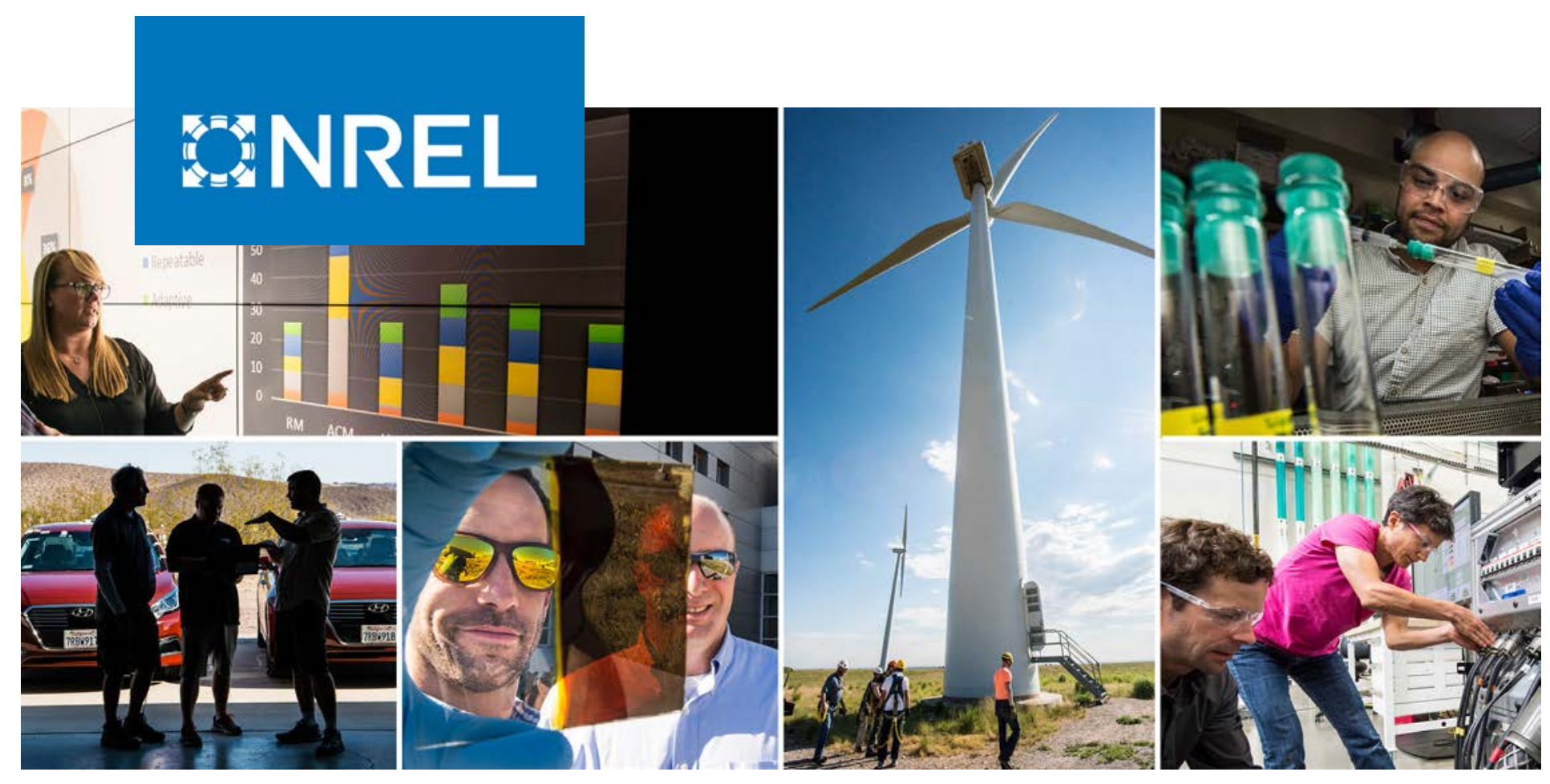

\title{
Gaining Insights in Loading Events for Wind Turbine Drivetrain Prognostics
}

\section{Preprint}

Pieter-Jan Daems, ${ }^{1}$ Yi Guo, ${ }^{2}$ Shawn Sheng, ${ }^{2}$ C. Peeters, ${ }^{1}$ P. Guillaume, ${ }^{1}$ and J. Helsen ${ }^{1}$

1 Vrije Universiteit Brussel

2 National Renewable Energy Laboratory

Presented at the Turbomachinery Technical Conference \& Exposition September $21-25,2020$

NREL is a national laboratory of the U.S. Department of Energy Office of Energy Efficiency \& Renewable Energy

Operated by the Alliance for Sustainable Energy, LLC

This report is available at no cost from the National Renewable Energy Laboratory (NREL) at www.nrel.gov/publications.

\section{Conference Paper}

NREL/CP-5000-76286

April 2020 


\title{
GNREL
}

\section{Gaining Insights in Loading Events for Wind Turbine Drivetrain Prognostics}

\section{Preprint}

\author{
Pieter-Jan Daems, ${ }^{1}$ Yi Guo, ${ }^{2}$ Shawn Sheng, ${ }^{2}$ C. Peeters, ${ }^{1}$ \\ P. Guillaume, ${ }^{1}$ and J. Helsen ${ }^{1}$ \\ 1 Vrije Universiteit Brussel \\ 2 National Renewable Energy Laboratory
}

\section{Suggested Citation}

Daems, Pieter-Jan; Yi Guo, Shawn Sheng, C. Peeters, P. Guillaume, and J. Helsen. 2020. Gaining Insights in Loading Events for Wind Turbine Drivetrain Prognostics:

Preprint. Golden, CO: National Renewable Energy Laboratory. NREL/CP-5000-76286.

https://www.nrel.gov/docs/fy20osti/76286.pdf

NREL is a national laboratory of the U.S. Department of Energy Office of Energy Efficiency \& Renewable Energy Operated by the Alliance for Sustainable Energy, LLC

This report is available at no cost from the National Renewable Energy Laboratory (NREL) at www.nrel.gov/publications.

Contract No. DE-AC36-08GO28308
Conference Paper NREL/CP-5000-76286 April 2020

National Renewable Energy Laboratory 15013 Denver West Parkway Golden, CO 80401 303-275-3000 • www.nrel.gov 


\section{NOTICE}

This work was authored [in part] by the National Renewable Energy Laboratory, operated by Alliance for Sustainable Energy, LLC, for the U.S. Department of Energy (DOE) under Contract No. DE-AC36-08GO28308. Funding provided by the U.S. Department of Energy Office of Energy Efficiency and Renewable Energy Wind Energy Technologies Office. The views expressed herein do not necessarily represent the views of the DOE or the U.S. Government. The U.S. Government retains and the publisher, by accepting the article for publication, acknowledges that the U.S. Government retains a nonexclusive, paid-up, irrevocable, worldwide license to publish or reproduce the published form of this work, or allow others to do so, for U.S. Government purposes.

This report is available at no cost from the National Renewable Energy Laboratory (NREL) at www.nrel.gov/publications.

U.S. Department of Energy (DOE) reports produced after 1991 and a growing number of pre-1991 documents are available free via www.OSTI.gov.

Cover Photos by Dennis Schroeder: (clockwise, left to right) NREL 51934, NREL 45897, NREL 42160, NREL 45891, NREL 48097, NREL 46526.

NREL prints on paper that contains recycled content. 


\section{GAINING INSIGHTS IN LOADING EVENTS FOR WIND TURBINE DRIVETRAIN PROGNOSTICS}

\author{
Pieter-Jan Daems \\ Department of Mechanical Engineering \\ Vrije Universiteit Brussel \\ Pleinlaan 2, 1050 Brussel (Belgium) \\ Email: pieter-jan.daems@vub.be
}

\author{
Y. Guo \\ S. Sheng \\ National Renewable Energy Laboratory \\ Golden, Colorado \\ C. Peeters \\ P. Guillaume \\ J. Helsen \\ Department of Mechanical Engineering \\ Vrije Universiteit Brussel \\ Pleinlaan 2, 1050 Brussel (Belgium)
}

\section{ABSTRACT}

Wind energy is one of the largest sources of renewable energy in the world. To further reduce the operations and maintenance $(O \& M)$ costs of wind farms, it is essential to be able to accurately pinpoint the root causes of different failure modes of interest. An example of such a failure mode that is not yet fully understood is white etching cracks (WEC). This can cause the bearing lifetime to be reduced to 5-10\% of its design value. Multiple hypotheses are available in literature concerning its cause. To be able to validate or disprove these hypotheses, it is essential to have historic high-frequency measurement data (e.g., load and vibration levels) available. In time, this will allow linking to the history of the turbine operating data with failure data. This paper discusses the dynamic loading on the turbine during certain events (e.g., emergency stops, run-ups, and during normal operating conditions). By combining the number of specific events that each turbine has seen with the severity of each event, it becomes possible to assess which turbines are most likely to show signs of damage.

\section{NOMENCLATURE}

LVRT Low voltage ride through

NREL National Renewable Energy Laboratory

MW megawatt

O\&M Operations and maintenance

SCADA Supervisory control and data acquisition

WEC White etching crack

\section{INTRODUCTION}

Over the last few years, the installed capacity of wind energy has increased drastically in Europe. To meet this increasing demand, an upscaling trend has been taking place: the turbines are increasing in size. It is hypothesized that by decreasing the number of machines needed per megawatt (MW), the operations and maintenance (O\&M) costs of the farms can be decreased [1]. This trend, however, also means that the failure of a single turbine causes larger energy losses for an equivalent downtime of the reference turbine. In order to be able to further reduce the O\&M costs, two aspects are essential. First, it is vital to be able to detect the degradation of the machine components before failure. In this way, predictive maintenance methodologies can be implemented, minimizing the downtime of the machine [2]. Second, it is important to be able to identify the root cause of the different failure modes, such that strategies can be found to mitigate the risk of these modes in future design iterations.

This paper focuses on the reliability at the level of the wind turbine gearbox. According to a National Renewable Energy Laboratory (NREL) study [3], the most frequent gearbox failure modes are related to the high-speed stage bearings (48\%) and the high-speed gears (18\%). For the former, white etching cracks (WECs) are the most common failure mode. WECs are crack networks that ultimately lead to macro-pitting in bearings [4]. Because of WECs, the bearing lifetime can drop to 5\%-10\% of their designed value [5] and increase the O\&M costs of wind turbine farms during their first years of operation.

Different hypotheses concerning the root cause of WECs ex- 
ist; however, no definitive conclusion has been found surrounding its root causes. In $[4,6]$, experimental studies were conducted to replicate WECs on a test rig and determine the influence of roller slip and lubricant additives. In this paper, the events for which roller slip can occur are targeted.

Wind turbines are subjected to different transient events. These transients can result in loading problems and unfavorable tribological conditions and will govern the design of the gearbox and bearings $[7,8]$. Examples of such events are the following:

1) Run-ups at low wind speed, as these can lead to damaging slip and smearing inside the bearings. In [9], it was observed that the roller slip density reached critical levels during a run-up before connecting the turbine to the grid.

2) Emergency stops and shock loads. During these events, it is known that the drivetrain is subject to torque reversals. These are introduced because of the dynamics of the gearbox (i.e., the first torsional mode) and result in sudden changes in the orientation of the loaded bearing zone [10]. During these reversals, loads will be applied to unloaded rollers, which can be detrimental for the drivetrain health. A possible trigger for these stops are, for example, low voltage ride through (LVRT), events where the generator momentarily loses connection to the electrical grid. This was also investigated in [9] and showed high roller slip losses when the unloaded rollers are accelerated after reconnecting to the grid.

Based on the observation that the roller slip plays a key role in the creation of WECs, different criteria have been proposed to attempt to predict the probability of WECs based on the operational history of the turbine. One is the accumulated friction energy, based on the sliding speed between the rolling element and the raceway and the normal load. Another similar metric is the power slip density. The common denominator between these two is that both require the ability to evaluate the loading conditions and the instantaneous slip. Therefore, it is vital to have detailed insights into the machine behavior, during both these transient events and the normal operating conditions. To this end, longterm high-frequency measurement data (e.g., load and vibration levels) should be available. In time, this will allow drawing correlations between the load history of the turbine and its failure record. Extensively monitoring the load and vibration levels is, however, unrealistic for entire fleets. Therefore, instrumenting all turbines with a minimal set of sensors and selecting a few turbines within the farm to instrument extensively is proposed. These are the so-called fleet leaders [11]. The other turbines within the farm are then assumed to have similar dynamic behavior during these different events of interest. By investigating in detail what happens at the level of the fleet leaders during these events, it becomes possible to define if these could be potentially harmful. By automatically these events on a farm-wide level and extrapolating the gained knowledge, it will become possible to obtain metrics of which turbines in the farm are most likely to show signs of damage.

To move toward this methodology, two major hurdles need to be overcome. The first one is related to the continuous acquisition of high-frequency measurement data at the level of the fleet leaders. Besides the acquisition, the device also needs to be capable of sending the useful information to a central location, where information of all turbines is available. To this end, two different strategies are possible. One methodology is to simply transmit all data to the central unit. Another option is to explore the viability of edge computing (i.e., processing part of the data at the level of the turbine and only transmit these results). The second challenge is related to the automatic processing of the data. For the fleet leaders, this includes having a set of algorithms available that can continuously process the incoming stream of high-frequency data, as the insights to be gained from the raw vibration and strain signals are often limited. Based on this, events of interest can be defined by the analyst. For the other turbines in the farm, these events should then be extracted automatically. In this way, statistics can be kept on how many events each turbine has seen, allowing correlations between these data and failure records in a final stage.

\section{METHODOLOGY}

This paper chooses to concentrate on the aspect of automatically processing the measured data. Within this context, two data sources are considered: Supervisory control and data acquisition (SCADA) data and strain gauge data measured at the level of the main speed shaft of the turbine. When data are continuously acquired, there is a need to separate steady-state behavior and transient behavior. Once a study of the machine loading in the different operating conditions has taken place, a damage metric can be attributed to different events and to steady-state operation. The latter is, however, beyond the scope of the paper, as the chosen metric is strongly problem dependent and is a field where extensive research has already taken place. Instead, the main goal is to present a framework where events are automatically detected and classified on a farm-wide level. This will be done in two steps.

First, data from the U.S. Department of Energy (DOE) 1.5MW turbine will be analyzed, as detailed instrumentation is available on this machine. Therefore, it can be used to gain detailed insights in what is happening for different similar machines for which only SCADA is available. To illustrate the developed algorithms, the forces at the level of the main shaft will be reflected to the carrier bearings. These forces can be in a last step condensed into a damage metric. Moreover, different events of interest are automatically identified using SCADA data of this turbine using a machine learning approach based on pattern mining and pattern matching. Second, one year of SCADA-1s data of 
an offshore wind farm will be analyzed. Because no force instrumentation is available on this wind farm, the focus here is solely on the detection of the different events. However, the algorithms implemented for assessing the bearing forces on the DOE turbine remain valid, as long as adequate design information of the gearbox is available and the needed measurement instrumentation is in place. The NREL data set is therefore used to demonstrate the feasibility of the different algorithms.

A schematic representation of the proposed methodology is shown in Figure 1. It can be seen that the general idea is to use the fleet leader to build a database for the events the turbine can see in the different ambient conditions (e.g., wind speeds and wind directions). This will allow an approximation of the loading that takes place during these conditions. Ideally, multiple fleet leaders are available to take into account wake effects throughout the farm. With this framework, it is possible to get an approximation of the accumulated damage for all turbines within the farm, allowing assessment of which machines are most likely to show damage.

\section{Load assessment}

As mentioned, there is use of the measurement data of the DOE 1.5-MW turbine located at NREL. Within the context of this paper, especially the instrumentation at the level of the main shaft are of interest. As the reliability of the bearings is central within this paper, there is the option to translate these forces to the bearing loads of the carriers using Equation 1. These equations can be retrieved by means of expressing the force and moment equilibrium for the system containing the main shaft and the carriers. The full derivation of the algorithm, as well as the made assumptions, can be found in [12]. The estimation of the tangential bearing forces $f_{t, i}$ requires having knowledge of several gearbox design parameters such as the tilting angle $\rho$, the mounting angle $\beta_{i}$, the shaft mass $m_{s h}$, and the distances from the main bearing to the centers of gravity of the main shaft $L_{s h}$, carrier $L_{c}$, and planetary gear $L_{p}$, respectively. $e_{i}$ are the deviation angles from the designed circumferential position (e.g., because of the planet-bearing clearance). For the gearbox under study, these parameters have been supplied by the manufacturer. These forces are cyclical in time, with $\Omega_{c}$ being the rotational frequency of the carrier axes. Because this force estimation requires continuous measuring of the applied moments to the drivetrain $M_{y}$ and $M_{z}$ and the applied torque $T_{i n}$, it is required to have strain information at the level of a drivetrain shaft. The unknown forces $f_{t, i}$ are then obtained on each time instance by minimizing the error terms $\varepsilon_{1}, \varepsilon_{2}$, and $\varepsilon_{3}$.

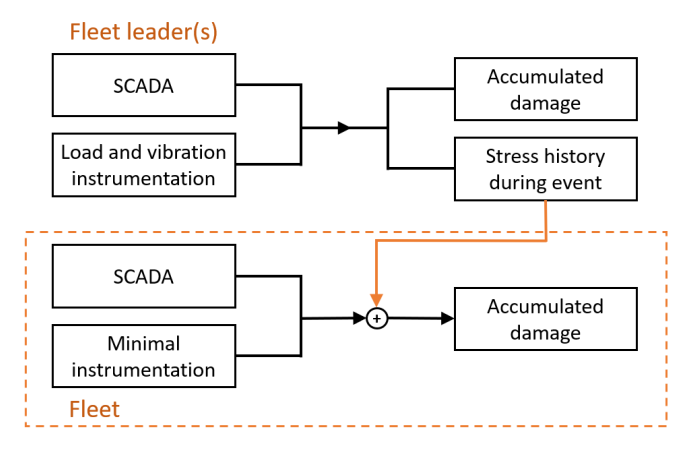

\section{FIGURE 1: BLOCK DIAGRAM SHOWING THE SUGGESTED METHODOLOGY.}

$$
\begin{aligned}
\varepsilon_{1}= & \frac{1}{2} m_{s h} g L_{s h} \cos (\rho)+\frac{M_{y}}{L_{c}}-\sum_{i=1}^{3} f_{t, i} \cos \left(\Omega_{c} t-\beta_{i}+e_{i}\right) \frac{L_{p}}{L_{c}} \\
& +\sum_{i=1}^{3} m_{p} g \sin \left(\Omega_{c} t+\beta_{i}+e_{i}\right)^{2} \cos (\rho) \frac{L_{p}}{L_{c}}+m g \cos (\rho) \\
\varepsilon_{2}= & \sum_{i=1}^{3} f_{t, i} d_{c}-T_{i n} \\
\varepsilon_{3}= & -\sum_{i=1}^{3} f_{t, i} \sin \left(\Omega_{c} t+\beta_{i}+e_{i}\right) L_{p}-M_{z} \\
& +\sum_{i=1}^{3} m_{p} g \sin \left(\Omega_{c} t+\beta_{i}+e_{i}\right) \cos \left(\Omega_{c} t+\beta_{i}+e_{i}\right) \cos (\rho) L_{p}
\end{aligned}
$$

\section{Detection of events of interest}

In this work, a methodology is investigated that can detect different events critical for drivetrain health automatically. It is assumed that the events that have to be detected are controlled; therefore, it is expected that the signatures in the time series are similar. As such, a machine learning approach based on pattern mining and matching is proposed.

Pattern mining algorithms have an objective of discovering all the different item sets in the given database. As this would result in $2^{N}$ possible subsets for a database with $\mathrm{N}$ items, this cannot be done using a brute force approach [13]. To this end, several algorithms have been proposed in literature to reduce the computational effort needed to calculate the frequent patterns, such as FP-growth, Prefixspan [14], and Freespan. In this work, there is use of a sequential pattern mining algorithm Prefixspan to extract the relevant patterns from the learned events. A sequential algorithm is clearly needed in this context to not lose the notion of the evolution of the event over time [15]. To move toward automatically detecting these events, a user has to manually label multiple in a set of training data. Based on this, the 
frequent patterns are extracted after binning the time series data. The less variable the event is, the fewer events need to be labeled by the user to identify the other similar events present in the training data automatically. This is implemented in the following way: First, it is determined whether or not a run-up or coast-down could be taking place. This can be done by observing if the bin data change in the bin within a certain time horizon. If this is the case, patterns are extracted and attempted to be matched with the reference set. To this end, the distance between two patterns is defined based on the difference in the number of samples in each bin. By summing this difference for all bins and by comparing this with a pre-imposed threshold based on the pattern length, similar time sequences can be matched with one another [16]. To make the performance less reliant on a well chosen subset of labeled events and to reduce the amount of needed user interaction, there is the option to only label a limited number of events manually and to implement an iterative procedure. In each subsequent iteration, more events will be able to be identified, allowing in turn to identify more patterns until no additional events are being identified. This makes the algorithm sufficiently robust in identifying events that have quite a large variability, such as a run-up at low wind speed, as will be demonstrated later on.

The performance of this algorithm will be showcased by processing one year of SCADA-1s data of an offshore wind farm. For the training stage of the algorithm, an analyst has selected several events. One month of one turbine is used to train the algorithm. Afterwards, transfer learning is used to process the other turbines in the farms. This transfer learning is achieved by extracting the set of representative patterns for the fleet leader and transferring these patterns to the other machines in the farm. In this way, high success rates $(98+\%)$ can be achieved.

The learned events consist of turbine starts and stops at both low and high wind speeds. The detection takes place using the SCADA-1s data, more specifically the gearbox speed and the pitch angles of the blades, as these have proven to produce the most representative patterns. For the stops at high wind speeds, status $\log$ s are used to further specify the reason the turbine stopped (e.g., yaw untwist, temperature alarm). These logs are not used earlier in the processing chain because they do not register stops at low wind speeds. Furthermore, multiple stops were often logged with these codes when only one physical stop had taken place. For the low wind speed stops, the wind speed and directions during the stop are extracted to account for the wake effects. By learning typical loading during these events on the fleet leader, extrapolation can happen to a farm-wide level, as seen in Figure 1. Within the context of matching the events of the fleet leader with the other turbines, pattern mining and matching also represent a powerful methodology, as it will allow the expression of the similarity between the different time series. Having multiple fleet leaders available in the farm will in practice allow reduction of the errors of approximation as it will be possible to learn over time which turbines will react more similarly to others in the different operating conditions. Because this approach learns how the turbine typically behaves during a start-up or coast-down in different wind conditions, this technique will remain valid in most cases even when faulty machine components are present. An exception to this might be faulty behavior at the level of the pitch motors.

\section{RESULTS \\ DOE 1.5-MW turbine}

As mentioned, it is of interest to be able to assess the bearing loading during different turbine operating conditions. As direct measurement of the bearing forces is not trivial, there is often the option to deduce these from the forces measured at the level of the main shaft using strain gauges. This is done using Equation 1. To guarantee that the turbine was running in steady-state, the previously discussed algorithm to detect events was used. In this way, different data sets with run-ups presented were identified, as can be seen in Figure 2. The dotted markers indicate the start of the event, whereas the starred markers indicate the end. The planet forces for one data set where the turbine is operating at rated power can be seen in Figure 3. The cyclical nature of this force as a result of the carrier rotation can clearly be seen. The forces of the three different planet bearings, indicated respectively in blue, orange, and green, are out of phase with respect to one another. These bearing forces can then in turn be translated to the roller forces. These can then be used in finite element simulations to reflect this to stress distributions in the material. As mentioned, this step is strongly problem dependent. For example, within the context of WECs, the influence of inclusions in the micro-structure is of interest because these are hypothesized to be critical spots for WEC initiation.

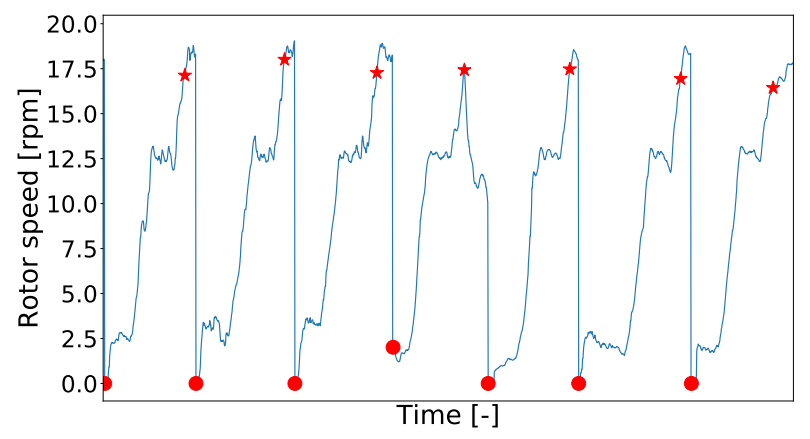

FIGURE 2: SEQUENCE OF AUTOMATICALLY LABELED RUN-UPS IN THE NREL TEST PROGRAM. 
Having accurate loading information at the level of the bearings is, however, not adequate, as current hypotheses list slip as one of the drivers in WEC formation. Therefore, we have to also be able to assess the amount of bearing slip during the different transients and operating conditions. In [17] and [18], an analytical approach to predict roller slip has been presented and validated using slip measurements for steady-state operation and different events such as LVRTs and emergency stops. These were then combined with load information to obtain metrics such as the cumulative friction energy and slip power density. It was shown that the main contributors to these metrics during steadystate operation are seen during the fast acceleration at the loadzone entry. For the transient events, up to $50 \%$ slip is seen.

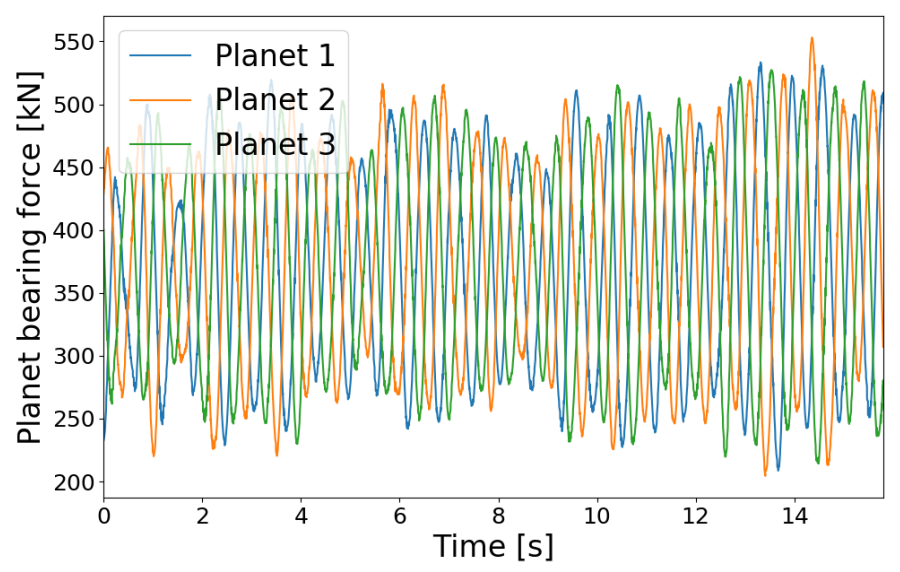

FIGURE 3: EVOLUTION OF THE BEARING FORCES OVER TIME.

\section{Offshore farm}

Using the described pattern mining approach, one year of SCADA data have been processed. An example of the results of the algorithm for one day of SCADA data is shown in Figure 4. The different colors indicate the different classes in which the algorithm has classified the events. A dotted marker is used to represent the start of the event, whereas the star indicates the end. Among the detected events, no significant amount of LVRTs has been detected, as the grid is more stable for these offshore farms than their onshore equivalent. By running this pipeline across a farm-wide level, insights are gained in which events each turbine sees throughout its lifetime.
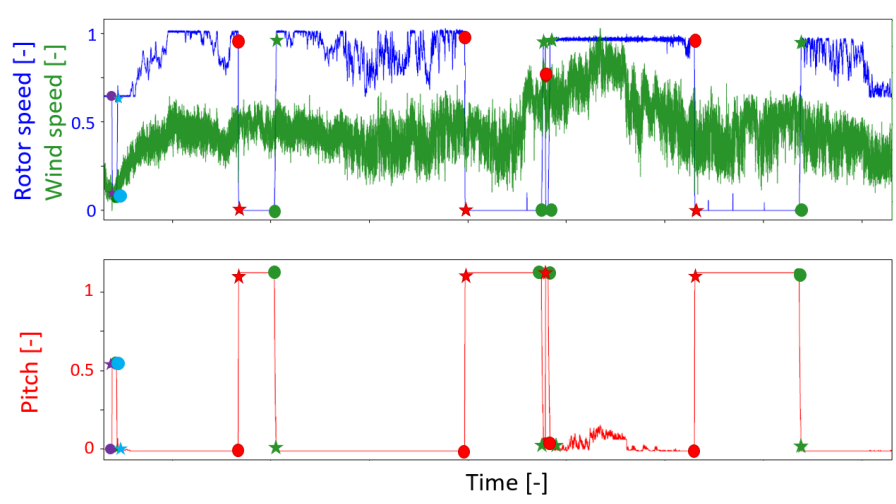

FIGURE 4: ONE DAY OF SCADA DATA PROCESSED WITH AUTOMATIC EVENT DETECTION. TEAL AND PURPLE INDICATE STARTS AND STOPS, RESPECTIVELY, AT WIND SPEEDS AROUND CUT-IN, AND RED AND GREEN INDICATE STOPS ABOVE CUT-IN.

By comparing the total number of events seen for each turbine, as well as the shape of the individual events, several observations can be made:

1) Some events such as run-ups at low wind speeds have a relatively large variability in their duration and general shape. This can be seen in Figure 5, where 150 run-ups of the same turbine are shown. These are synchronized to coincide at half of the rated speed. Based on this observation, it is expected that the calculated damage metrics (e.g., accumulated friction energy) during these events are also significantly different, showing the need for better understanding the sources of this variability and learning under which conditions these metrics become significantly higher to be able to avoid these when possible.

Furthermore, it can be seen that there are a couple of events where the wind speed starts decreasing during the run-up, resulting in the turbine doing a coast-down again. Making a distinction between these different types is important, as there will be significant slip and loading at the bearings at the moment when the turbine will connect to the grid. At the moment of grid connection, there will be large slip combined with large loading, meaning that these events are potentially more critical for the health of the drivetrain.

2) There is a significant difference in the number of events that each turbine within the farm sees. This is shown in Figure 6 , where the total number of run-ups at low wind can be seen for a subset of 20 turbines within the farm. No trivial correlations were found between the number of events the turbines see and the layout of the farm. Besides the number 


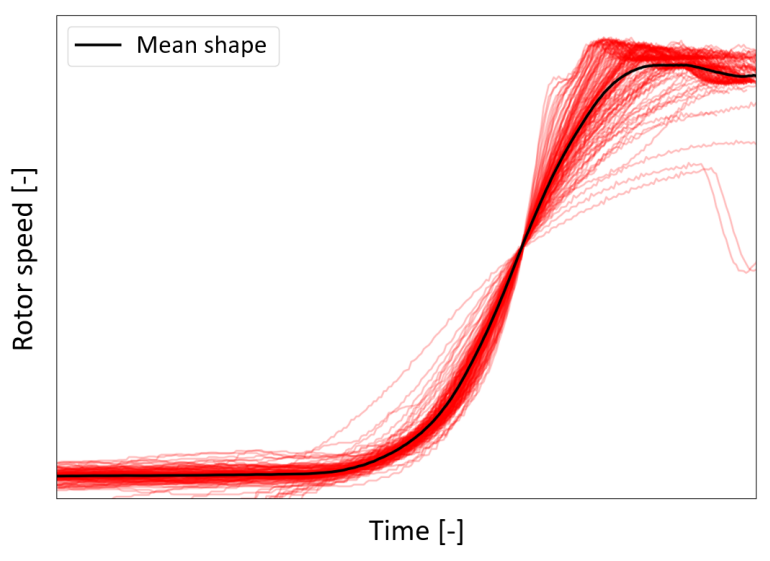

FIGURE 5: VARIABILITY IN SHAPE BETWEEN DIFFERENT RUN-UPS AT LOW WIND SPEED. (AXES NORMALIZED FOR CONFIDENTIALITY)

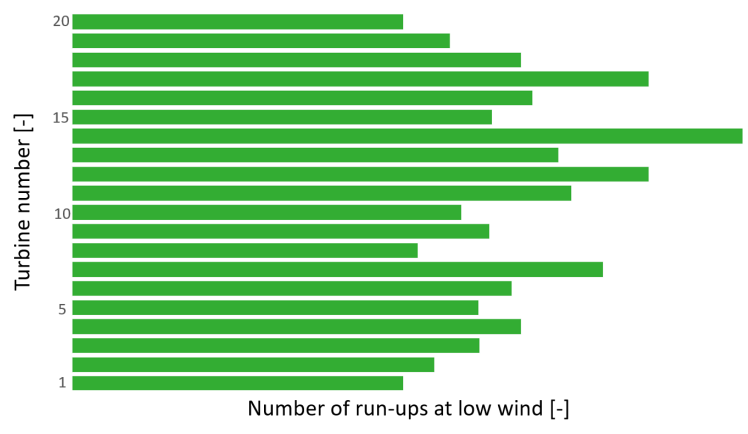

\section{FIGURE 6: NUMBER OF EVENTS SEEN FOR THE DIFFERENT TURBINES IN THE FARM. (AXES NORMALIZED FOR CONFIDENTIALITY)}

of events strongly varying, the percentage of run-ups where grid connection took place is significantly different across the farm.

Based on the two previous observations, it is clear that the two metrics proposed in the literature for WEC formation (i.e., accumulated frictional energy and power slip density) can vary for the different turbines in the farm. It is also important that these events at low wind speeds cannot be detected using conventional SCADA 10-minute and status log data. The proposed methodology therefore allows a ranking of which turbines are most likely to show WECs in the farm. This combined with health metrics obtained with condition monitoring allows in turn to get objective information about which turbines to inspect.

\section{CONCLUSIONS AND FUTURE WORK}

This paper has presented an approach to automatically detect different events of interest on a farm-wide level. To this end, a first analysis was done on the DOE 1.5-MW turbine. On this turbine, events were extracted using SCADA data, and the bearing forces were estimated using strain gauge measurements at the main shaft. This was done such that each operating condition could be attributed with a damage metric. After this study on this single turbine, SCADA data of an offshore wind farm were analyzed. It was shown that the number of these events can strongly vary across the different turbines of the farm. As these can be critical for drivetrain health, these insights are vital to be able to start seeing correlations between failure data and the events seen by the turbine throughout its lifetime.

The future work consists of instrumenting several turbines on the offshore wind farm to be able to work with representative loading for this turbine and to be able to explore the presented fleet leader concept in more detail. On the other hand, finite element models will be developed to predict what is happening at the level of the material. These steps will allow validation in more detail to the different hypotheses concerning the correlations between WECs and the complex operating conditions of wind turbines.

\section{ACKNOWLEDGMENT}

The authors acknowledge the financial support of the MaDurOS program from VLAIO (Flemish Agency for Innovation and Entrepreneurship) and SIM (Strategic Initiative Materials) through project SBO MaSiWEC (HBC.2017.0606). The authors thank their partners for delivering the monitoring data. This work was authored [in part] by the National Renewable Energy Laboratory, operated by Alliance for Sustainable Energy, LLC, for the U.S. Department of Energy (DOE) under Contract No. DE-AC36-08GO28308. Funding provided by the U.S. Department of Energy Office of Energy Efficiency and Renewable Energy Wind Energy Technologies Office. The views expressed in the article do not necessarily represent the views of the DOE or the U.S. Government. The U.S. Government retains and the publisher, by accepting the article for publication, acknowledges that the U.S. Government retains a nonexclusive, paid-up, irrevocable, worldwide license to publish or reproduce the published form of this work, or allow others to do so, for U.S. Government purposes.

\section{REFERENCES}

[1] Helsen, J., Vanhollebeke, F., Vandepitte, D., and Desmet, W., 2012. "Some trends and challenges in wind turbine upscaling". In Proceedings of ISMA International Conference On Noise And Vibration 2012, Vol. 6, pp. 4345-4359. 
[2] Verstraeten, T., Marulanda, F. G., Peeters, C., Daems, P.J., Nowé, A., and Helsen, J., 2019. "Edge computing for advanced vibration signal processing". In Surveillance, Vishno and AVE conferences, Hal archives ouvertes, pp. 234-240.

[3] Sheng, S., 2017. Wind turbine gearbox reliability database, operation and maintenance research update. Report NREL/PR-5000-68347, National Renewable Energy Laboratory.

[4] Gould, B., and Greco, A., 2015. "The influence of sliding and contact severity on the generation of white etching cracks". Tribology Letters, 60(2), p. 29.

[5] Evans, M., 2012. "White structure flaking (wsf) in wind turbine gearbox bearings: effects of butterflies and white etching cracks (wecs)". Materials Science and Technology, 28(1), pp. 3-22.

[6] Gould, B., Demas, N. G., Pollard, G., Rydel, J. J., Ingram, M., and Greco, A. C., 2019. "The effect of lubricant composition on white etching crack failures". Tribology Letters, 67(1), p. 7.

[7] Helsen, J., Guo, Y., Keller, J., and Guillaume, P., 2016. "Experimental investigation of bearing slip in a wind turbine gearbox during a transient grid loss event". Wind Energy, 19(12), pp. 2255-2269.

[8] Evans, M.-H., 2016. "An updated review: white etching cracks (wecs) and axial cracks in wind turbine gearbox bearings". Materials Science and Technology, 32(11), pp. 1133-1169.

[9] Vaes, D., Clement, P., and Stadler, K., 2017. "How does the application of actual standards and guidelines contribute to robust bearing solution in multi-mw wind turbine gearboxes". In Conference for Wind Power Drives (CWD), Vol. 3, pp. 43-58.

[10] Helsen, J., Guo, Y., and Keller, J., 2018. "Gearbox highspeed-stage bearing slip induced by electric excitation in a test facility". Wind Energy, 21(11), pp. 1191-1201.

[11] Weijtens, W., Noppe, N., Verbelen, T., Iliopoulos, A., and Devriendt, C., 2016. "Offshore wind turbine foundation monitoring, extrapolating fatigue measurements from fleet leaders to the entire wind farm". Journal of Physics: Conference Series, 753(9).

[12] Guo, Y., Keller, J., and LaCava, W., 2015. "Planetary gear load sharing of wind turbine drivetrains subjected to nontorque loads". Wind Energy, 18(4), pp. 757-768.

[13] Feremans, L., Cule, B., Devriendt, C., Goethals, B., and Helsen, J., 2017. "Pattern mining for learning typical turbine response during dynamic wind turbine events". In ASME 2017 International Design Engineering Technical Conferences and Computers and Information in Engineering Conference, American Society of Mechanical Engineers Digital Collection.

[14] Pei, J., Han, J., Mortazavi-Asl, B., Pinto, H., Chen, Q.,
Dayal, U., and Hsu, M.-C., 2001. "Prefixspan: Mining sequential patterns efficiently by prefix-projected pattern growth". In Proceedings 17th International Conference on Data Engineering, IEEE, pp. 215-224.

[15] Daems, P., Feremans, L., Verstraeten, T., Cule, B., Goethals, B., and Helsen, J., 2019. "Fleet-oriented pattern mining combined with time series signature extraction for understanding of wind farm response to storm conditions". In World Conference for Condition Monitoring (WCCM), Springer.

[16] Daems, P., Peeters, C., Verbeke, J., and Helsen, J., 2018. "Automatic detection of events critical for drivetrain health and lifetime from long-term field measurements". In Conference for Wind Turbine Drivetrains (CWD), Vol. 4.

[17] Vaes, D., Keller, J., Tesini, P., and Guo, Y., 2018. "Roller sliding in wind turbine gearbox high-speed-shaft bearings". In Conference for Wind Power Drives (CWD), Vol. 4, pp. 1-7.

[18] Vaes, D., Guo, Y., Tesini, P., and Keller, J., 2019. "Investigation of roller sliding in wind turbine gearbox highspeed-shaft bearings". In National Renewable Energy Lab: Technical report, pp. 1-28. 